\title{
Traumatic brain injury in high versus low falls in young children and adolescents: a retrospective analysis
}

\author{
Angela Bandte, MD, ${ }^{1}$ Klaus Püschel, MD, ${ }^{1}$ and Kara Krajewski, MD² \\ ${ }^{1}$ Institute for Forensic Medicine and ${ }^{2}$ Department of Neurosurgery, University Medical Center Hamburg-Eppendorf, Hamburg, \\ Germany
}

OBJECTIVE So-called low falls are gaining interest in traumatology. To date, there are no studies on low versus high falls in the pediatric population. The aim of the current analysis was to characterize the symptoms, diagnostics, and injuries associated with high versus low falls and to examine the effect of fall height on injuries and outcome in the context of current guideline-based recommendations.

METHODS After obtaining study approval from the local ethics committee, the authors reviewed the data for patients ages 5-17 years who had been consecutively treated at either hospital associated with the University Medical Center Hamburg in the period from January 2009 to August 2014 for diagnoses including traumatic brain injury (TBI). Retrospective analysis of the electronic patient charts was performed to obtain data on demographics; accident mechanism; initial neurological status with respect to consciousness, symptoms, and Glasgow Coma Scale score; radiological studies; diagnoses; length of stay; all intracranial procedures; and Glasgow Outcome Scale (GOS) score.

RESULTS Sixty-five fall-related TBls among 380 patients were identified; 26 patients fell from a height of $3 \mathrm{~m}$ or more and 28 fell from a height under $3 \mathrm{~m}$ (height undocumented in 11 cases). Patients who fell from $\geq 3 \mathrm{~m}$ were 22 times more likely than those who fell from $<3 \mathrm{~m}$ to undergo spiral CT studies in the emergency room $(p=0.05)$. In addition, there was a 7.4 times greater chance of undergoing cranial CT $(p=0.02)$. There were significantly more noncerebral injuries requiring surgery in patients who fell from $\geq 3 \mathrm{~m}$ versus those who fell from $<3 \mathrm{~m}(p=0.007)$. There was no difference in the frequency of neurosurgical procedures performed between low and high groups. Follow-up ranged from 0 to 92 months (mean 12.5 months, median 0 months). There was no significant difference in good (GOS score 4-5) and poor (GOS score 1-3) outcomes between high and low falls $(p=0.208)$.

CONCLUSIONS Low falls can be associated with intracranial hemorrhages requiring ICU monitoring and/or surgery. The authors encourage intensive monitoring and CT scans based on clinical presentation, not on fall height.

https://thejns.org/doi/abs/10.3171/2018.2.PEDS17714

KEYWORDS children; traumatic brain injury; falls; trauma

$\mathrm{F}$ ALLS most commonly occur as accidents. In young children, they can be related to child abuse. In older children, they can be related to suicide attempts. Clinicians in Germany base their treatment decisions on the guidelines published by the national Association of the Scientific Medical Societies in Germany. The trauma guidelines are an interdisciplinary effort coordinated and led by the German Society of Trauma Surgery and were recently updated (http://www.awmf.org/en/clinical-practice-guide lines/detail/11/012-019.html). According to the guidelines, falls from a height over $3 \mathrm{~m}$ should be treated in the trauma room. Cranial CT scans may be necessary for "high-velocity trauma," which is defined as a fall from a height over $6 \mathrm{~m}$, among other things (e.g., traffic accidents). Specific guidelines for children are currently being developed.

So-called low falls are gaining interest, especially in the geriatric population. The literature regarding falls, especially those from low heights, is sparse. In falls from greater heights, there is some differentiation with respect to the occurrence of traumatic brain injury (TBI). Below a

ABBREVIATIONS DAI = diffuse axonal injury; GCS = Glasgow Coma Scale; GOS = Glasgow Outcome Scale; TBI = traumatic brain injury.

SUBMITTED December 14, 2017. ACCEPTED February 27, 2018.

INCLUDE WHEN CITING Published online June 1, 2018; DOI: 10.3171/2018.2.PEDS17714. 
10-m height, it is deemed unlikely that the body position changes during the fall; therefore, TBI occurrence is affected by whether someone falls head first or not. Between 10 and $25 \mathrm{~m}$, the occurrence of TBI decreases as body position is altered during falls with a tendency to land feet first. From a height exceeding $25 \mathrm{~m}$, the landing position has less influence on TBI occurrence as forces are strong enough to cause TBI even when a person lands feet first. For falls under $25 \mathrm{~m}$, non-TBI injuries are an especially important determinant of outcome. However, few studies have further analyzed falls below $10 \mathrm{~m} \cdot{ }^{1,9,10,11}$ An analysis of high versus low falls in the pediatric population specifically is lacking.

With mounting evidence of an increased risk of cancers in children associated with CT exposure, ${ }^{7}$ it becomes especially important to weigh the indication for such imaging studies very carefully. Thus, the aim of this analysis was to characterize the symptoms, diagnostics, and injuries associated with high versus low falls and to examine the effect of fall height on injuries and outcome in the context of current guideline-based recommendations.

\section{Methods}

Study approval from the local ethics committee was obtained. Patients ages 5-17 years who had been consecutively treated at either hospital associated with the University Medical Center Hamburg in the period from January 2009 to August 2014 for diagnoses including TBI were included in this study. Retrospective analysis of the electronic patient charts was performed to obtain data on the following parameters: age; sex; activity leading to the accident; accident mechanism; initial neurological status with respect to consciousness, symptoms, and Glasgow Coma Scale (GCS) score; radiological studies; diagnoses including skull fractures, intracranial hemorrhage, and nonneurological injuries requiring surgery; length of stay in the hospital and ICU; all intracranial procedures including invasive intracranial pressure monitoring; and neurological status (Glasgow Outcome Scale [GOS] score) on discharge or the latest follow-up. All data were accessed and analyzed according to current data protection regulations.

Descriptive analysis was performed with Microsoft Excel and SPSS (SPSS Inc.). Statistical analysis was per- formed with SPSS. Logistic regression was used to calculate correlations between risk factors and injuries. Parameters were analyzed with uni- and multivariate logistic regression. A p value $<0.05$ was regarded as significant.

\section{Results}

Three hundred eighty children (162 [42.6\%] female and 218 [57.4\%] male) met the inclusion criteria within the study time frame. Mean age was 11.9 years. Sixty-five patients had suffered a fall-related TBI. Most of the remaining 315 cases were sports-related injury, including bicycling, soccer, and horseback riding accidents as well as traffic accidents. These patients with injuries unrelated to TBI served as a reference group for a portion of our analyses.

Among the 65 patients who suffered falls, 26 fell from a height of $3 \mathrm{~m}$ or higher and 28 fell from a height under $3 \mathrm{~m}$. In 11 patients the fall height was not documented, so these cases were eliminated from further analyses. Among the 54 patients with documented fall heights, falls ranged from 0.4 to $12.5 \mathrm{~m}$ (median $2.65 \mathrm{~m}$ ). The mean age among this group was 9.2 years, and 59\% were male and $41 \%$ were female. Falls were the result of climbing/play (e.g., trees, jungle gyms) in 22 cases and falling down/off objects (e.g., stairs, bunk beds) in 31 cases. One case was an attempted suicide.

There was no difference between low and high fall cases with respect to concussion-related symptoms on admission, such as unconsciousness, headaches, nausea, amnesia, and focal neurological deficits (data not shown); however, low falls were associated with a 4.8 times higher risk of vomiting on admission compared to non-fall-related TBIs ( $p=0.02,95 \%$ CI $1.8-13)$.

Initial GCS scores were documented for 26 patients (Fig. 1). There was no significant difference between highand low-fall cases with respect to a GCS score $>9$ versus $<9(\mathrm{p}=0.23$, Fisher's exact test).

Patients who fell from a height $\geq 3 \mathrm{~m}$ were 22 times more likely than those who fell from $<3 \mathrm{~m}$ to undergo spiral CT studies in the emergency room $(\mathrm{p}=0.05,95 \% \mathrm{CI}$ 2.6-188). In addition, there was a 7.4 times greater chance of undergoing cranial CT ( $\mathrm{p}=0.02,95 \%$ CI 2-27.3). All CTs in children were performed with age-adapted lowdose pediatric protocols (Table 1).

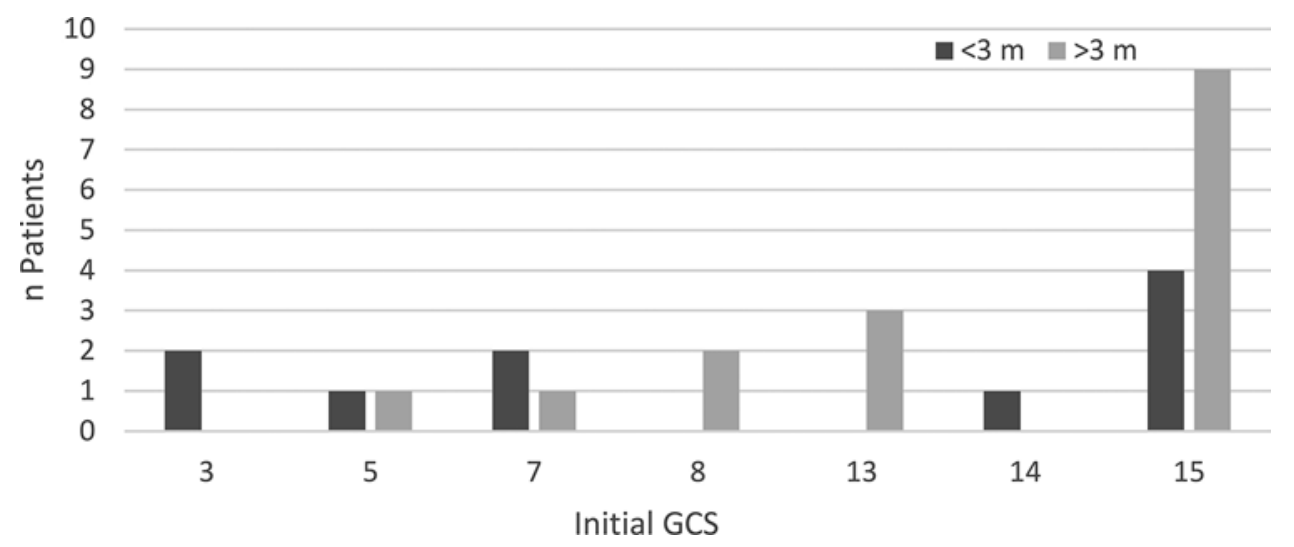

FIG. 1. The initial GCS score distribution for 26 patients, according to fall height. 
TABLE 1. Imaging studies performed according to fall height

\begin{tabular}{|c|c|c|c|c|c|c|c|c|}
\hline \multirow[b]{3}{*}{ Fall Height } & \multicolumn{8}{|c|}{ Imaging Study } \\
\hline & \multicolumn{2}{|c|}{$\begin{array}{c}\text { Spiral } \\
\text { CT }\end{array}$} & \multicolumn{2}{|c|}{$\begin{array}{c}\text { Cranial } \\
\text { CT }\end{array}$} & \multicolumn{2}{|c|}{$\begin{array}{c}\text { Facial } \\
\text { Bone CT }\end{array}$} & \multicolumn{2}{|c|}{$\begin{array}{c}\text { Cranial } \\
\text { MRI }\end{array}$} \\
\hline & No & Yes & No & Yes & No & Yes & No & Yes \\
\hline$\geq 3 m(n=26)$ & 14 & $11^{*}$ & 4 & $21 \dagger$ & 23 & 2 & 24 & 1 \\
\hline$<3 \mathrm{~m}(\mathrm{n}=28)$ & 28 & $1^{*}$ & 17 & $12 \dagger$ & 29 & 0 & 24 & 5 \\
\hline
\end{tabular}

$\mathrm{n}=$ number of patients.

Low-dose CT protocols are standard for patients ages 18 years and under.

* $O R=22$ for $\geq 3 \mathrm{~m}$ versus $<3 \mathrm{~m}(\mathrm{p}=0.05)$.

$\dagger O R=7.4$ for $\geq 3 \mathrm{~m}$ versus $<3 \mathrm{~m}(\mathrm{p}=0.02)$.

The frequency and types of hemorrhage as diagnosed via imaging are shown in Table 2. There was no difference between the type or frequency of hemorrhage and fall height $(p=0.77)$. Neither was there any difference in the frequency of neurosurgical procedures performed between low and high groups (data not shown). We did observe that the patients who required neurosurgical intervention after a low fall had fallen directly onto their head.

There were significantly more noncerebral injuries requiring surgery in patients with falls $\geq 3 \mathrm{~m}$ versus those $<$ $3 \mathrm{~m}(\mathrm{p}=0.007$; Table 3$)$.

There was no significant difference between high and low falls with respect to length of stay in the hospital and ICU (data not shown).

The GOS score was available for 52 patients. For 56\% of the patients, the GOS score was available at discharge from the hospital. Follow-up ranged from 0 to 92 months (mean 12.5 months, median 0 months). Ninety-four percent of the patients had a GOS score of 5. One death occurred (GOS score 1; described below), as did one case of severe disability (GOS score 3 ) and one case of moderate disability (GOS score 4), all of which were associated with high falls. After dichotomizing outcomes into good (GOS score 4-5) and poor (GOS score 1-3) categories, we found no significant difference in outcome between high and low falls ( $p=0.208$, Fisher's exact test).

In the one case of death, the patient was a 13-year-old male who had an accidental fall from approximately 3-4 $\mathrm{m}$ and suffered an isolated severe TBI including multiple skull fractures with an initial GCS score of 5. Despite maximal conservative therapy, he died within several hours of presentation in the ICU as a result of brainstem herniation.

\section{Discussion}

We analyzed low versus high falls in the pediatric population for the first time. Clinical presentation did not differ between the groups with respect to symptoms and initial GCS score. Intracranial hemorrhage and neurosurgical interventions occurred equally in the two groups. There was indirect evidence of a more severe clinical presentation with respect to vomiting on admission for low falls compared to non-fall-related TBIs. As in other series in adults, our data showed a statistically significant higher risk of noncerebral injury requiring surgery associated with high falls, although outcome was similar in the two fall groups.
TABLE 2. Intracranial hemorrhage distribution according to fall height

\begin{tabular}{lcc}
\hline & \multicolumn{2}{c}{ Fall Height } \\
\cline { 2 - 3 } Type of Hemorrhage & $<3 \mathrm{~m}$ & $\geq 3 \mathrm{~m}$ \\
\hline Subdural hematoma & 1 & 1 \\
\hline Traumatic SAH & 0 & 2 \\
\hline Epidural hematoma & 1 & 0 \\
\hline Intracerebral hematoma & 1 & 1 \\
\hline Multiple hemorrhages & 1 & 1 \\
\hline
\end{tabular}

SAH = subarachnoid hemorrhage.

A fall from a height $\geq 3 \mathrm{~m}$ was associated with a significantly increased risk of undergoing cranial or spiral $\mathrm{CT}$, compared to a fall from $<3 \mathrm{~m}$ in height. In accordance with the 2011 German polytrauma guidelines, this finding was to be expected as CT scans were officially recommended as an optional indication for falls from $3 \mathrm{~m}$ or higher. However, the necessity of scans must be questioned as clinical presentation and initial GCS scores were similar in our two groups. One must question whether the scans were a product of the fear of medicolegal repercussions under the influence of clear guideline recommendations.

A large study in adults with low falls defined as $<2$ $\mathrm{m}$ showed that such falls accounted for nearly half of all admissions and were associated with older, more chronically ill patients. Overall, there were fewer severely injured patients, less ICU time, and fewer deaths (except for sports-related trauma) compared to those with high falls as well as those with motor vehicle accidents, sports injuries, or assault injuries. ${ }^{4}$ These findings are difficult to apply to the pediatric population. In contrast, Helling et al. examined findings in adult patients who had fallen from less than $6 \mathrm{~m} .{ }^{3}$ The most commonly sustained injury was TBI, with $35 \%$ of the included patients suffering such an injury. Head injury was also the most common cause of death. It is difficult to compare our findings to data in the aforementioned study as its authors analyzed all falls for TBI, whereas we analyzed all TBI for falls. However, their study demonstrates the potential severity of low falls in the adult population.

As mentioned above, vomiting on initial admission occurred more frequently in low-fall cases than in other non-fall-related TBIs in the present study. Vomiting as-

TABLE 3. Incidence of concomitant noncerebral injury requiring surgery, according to fall height

\begin{tabular}{lrrr}
\hline & \multicolumn{2}{c}{ Fall Height } & \\
\cline { 2 - 3 } \multicolumn{1}{c}{ Parameter } & $<3 \mathrm{~m}$ & $\geq 3 \mathrm{~m}$ & Total \\
\hline Additional injury requiring surgery & & & \\
\hline No & 27 & 15 & 42 \\
\hline Yes & 2 & 10 & 12 \\
\hline Total & 29 & 25 & 54 \\
\hline
\end{tabular}

$p=0.007$, Fisher's exact test. 
sociated with TBI is considered a symptom of elevated intracranial pressure, which can be related to intracranial hemorrhage or diffuse axonal injury (DAI). Dunning et al. showed that emesis was not a good predictor of substantial TBI. ${ }^{2}$ While the incidence of DAI could not be assessed in the current study, intracranial hemorrhage did occur in our low-fall group. We do not have the patient numbers required to calculate risk analyses. Taken together, however, our data do not support the assumption that low falls do not require interdisciplinary trauma room care and/or may not cause serious injury requiring immediate surgery.

Thus, it was not immediately apparent why the German trauma guidelines changed their definition of highvelocity trauma from falls above $3 \mathrm{~m}$ to falls above $6 \mathrm{~m}$, ultimately changing the threshold for a CT scan. Kohn et al., who were cited in the new 2016 trauma guidelines, found that $9.4 \%$ of patients with falls from over $6 \mathrm{~m} \mathrm{had}$ severe injuries, which were defined as those requiring ICU treatment or immediate surgical treatment. ${ }^{5}$ Further, Yagmur et al., who were also cited, posited that death after falls was associated with a mean height of $9 \mathrm{~m}$ in adults. ${ }^{12}$ However, this group also found that the mean fall height in children under the age of 15 years who had died from the fall was $4 \mathrm{~m}^{12}$

There is growing evidence that CT scans are overused in the pediatric population and extremely harmful. An analysis of over 42,000 children who had presented with isolated loss of consciousness after TBI and a GCS score 13-15 revealed a very low risk of "clinically important TBI" requiring surgery, intubation, or hospitalization for 2 or more nights or resulting in death, and thus CT was not recommended. ${ }^{6}$

More importantly, Miglioretti et al. found an increased risk of solid cancers and leukemia in children associated with cranial CT exposure. These data indicate a palpable risk with unnecessary CT scans. However, a calculated risk model incorporating both TBI rates and cancer rates in the same population is needed to complete the picture.

The present data seem to provide arguments to override these risks. Our goal is not to increase CT rates, but rather to increase awareness of the potential serious injury associated with low falls in children. Schnadower et al. provided solid recommendations based on several metaanalyses and cited an indication to discharge an asymptomatic child without further observation if falls were below $1 \mathrm{~m} .{ }^{8}$ Our observation of a relation between lows falls with intracranial hemorrhage requiring surgery and an injury mechanism of falling directly onto the head must be further analyzed in larger studies with odds ratio models. We believe that regardless of the fall height, clinical presentation should dictate further diagnostics and management. We urge clinicians not to have a false sense of security when low fall heights are reported, especially if the patient has fallen directly onto the head.

As the present study showed a 22 times greater risk of cranial CT in falls from a height $\geq 3 \mathrm{~m}$, in accordance with recommendations from the guidelines in place at the time, it will be interesting to see if this rate is further reduced over the next 5 years in accordance with the new trauma guidelines, especially in light of the emerging data on CT exposure-related cancer risk. The new children's hospital at the University Medical Center in Hamburg, Germany, has only an MRI scanner; CT will be reserved for interdisciplinary trauma room emergencies. Age-adapted lowdose protocols have been the standard for our pediatric patients for several years. Our goal is to reduce unnecessary exposure without compromising patient safety.

Limitations of this study include its retrospective design. There was a surprising lack of documentation on the initial GCS score, for example. Fortunately, documentation of the initial GCS score is now an official part of the new German trauma guidelines, which will help us to better examine TBI in future studies. We also aim to extend the follow-up period in future studies and include neuropsychological assessments as we believe that the GOS is too coarse a measure of outcome, especially in children. Further, although we were able to provide a good regional overview of TBI in children in the greater Hamburg area, because the majority of pediatric patients with TBI are treated at one of our university locations, the sample size was ultimately too small to provide the necessary statistical power behind our findings. In addition, the results may not generally apply to the rest of Germany since the cohort was regional. However, we were able to identify key points for future multicenter studies.

\section{Conclusions}

The height of a fall does have influence on guidelines and clinical decisions. In comparison to the 2011 version, the 2016 version of the polytrauma guidelines indicates that a fall from over $6 \mathrm{~m}$ constitutes a high-velocity trauma, which may require CT diagnostics. However, our results suggested that low falls can be associated with intracranial hemorrhages requiring ICU monitoring and/ or surgery. Despite falling from a lower height, a patient can suffer severe injuries, and all management decisions should be based strictly on the clinical condition of the patient. If in doubt, the patient should be monitored closely even if the fall occurred from a low height.

\section{Acknowledgments}

We thank Ms. Lezius at the Institute for Medial Biometrics and Epidemiology for her support with statistical analyses. Prof. Sommerfeldt deserves our gratitude for his assistance with the data collection from Altona Children's Hospital. And we thank Dr. Hessler and the entire Riding Safety Team for their input and support.

\section{References}

1. Atanasijevic TC, Savic SN, Nikolic SD, Djoki VM: Frequency and severity of injuries in correlation with the height of fall. J Forensic Sci 50:608-612, 2005

2. Dunning J, Batchelor J, Stratford-Smith P, Teece S, Browne J, Sharpin C, et al: A meta-analysis of variables that predict significant intracranial injury in minor head trauma. Arch Dis Child 89:653-659, 2004

3. Helling TS, Watkins M, Evans LL, Nelson PW, Shook JW, Van Way CW: Low falls: an underappreciated mechanism of injury. J Trauma 46:453-456, 1999

4. Kennedy RL, Grant PT, Blackwell D: Low-impact falls: demands on a system of trauma management, prediction of outcome, and influence of comorbidities. J Trauma 51:717-724, 2001 
5. Kohn MA, Hammel JM, Bretz SW, Stangby A: Trauma team activation criteria as predictors of patient disposition from the emergency department. Acad Emerg Med 11:1-9, 2004

6. Lee LK, Monroe D, Bachman MC, Glass TF, Mahajan PV, Cooper A, et al: Isolated loss of consciousness in children with minor blunt head trauma. JAMA Pediatr 168:837-843, 2014

7. Miglioretti DL, Johnson E, Williams A, Greenlee RT, Weinmann S, Solberg LI, et al: The use of computed tomography in pediatrics and the associated radiation exposure and estimated cancer risk. JAMA Pediatr 167:700-707, 2013

8. Schnadower D, Vazquez H, Lee J, Dayan P, Roskind CG: Controversies in the evaluation and management of minor blunt head trauma in children. Curr Opin Pediatr 19:258264, 2007

9. Teh J, Firth M, Sharma A, Wilson A, Reznek R, Chan O: Jumpers and fallers: a comparison of the distribution of skeletal injury. Clin Radiol 58:482-486, 2003

10. Thierauf A, Preuss J, Lignitz E, Madea B: Retrospective analysis of fatal falls. Forensic Sci Int 198:92-96, 2010

11. Türk EE, Tsokos M: Pathologic features of fatal falls from height. Am J Forensic Med Pathol 25:194-199, 2004

12. Yagmur Y, Güloğlu C, Aldemir M, Orak M: Falls from flat- roofed houses: a surgical experience of 1643 patients. Injury 35:425-428, 2004

\section{Disclosures}

The authors report no conflict of interest concerning the materials or methods used in this study or the findings specified in this paper.

\section{Author Contributions}

Conception and design: all authors. Acquisition of data: all authors. Analysis and interpretation of data: Krajewski, Bandte. Drafting the article: Krajewski. Critically revising the article: Krajewski, Bandte. Reviewed submitted version of manuscript: Krajewski. Approved the final version of the manuscript on behalf of all authors: Krajewski. Statistical analysis: Krajewski. Administrative/technical/material support: Püschel. Study supervision: Püschel.

\section{Correspondence}

Kara Krajewski: University Medical Center SH Campus Lübeck, Germany.karaleigh.krajewski@uksh.de. 